\title{
Weak point disorder in strongly fluctuating flux-line liquids
}

\author{
Panayotis Benetatos ${ }^{1}$ and M. Cristina Marchetti ${ }^{2}$ \\ ${ }^{1}$ Hahn-Meitner-Institut, Abteilung Theoretische Physik (SF5), \\ Glienicker Str. 100, D-14109, Berlin, Germany \\ 2 Physics Department, Syracuse University, \\ Syracuse, NY, 13244, USA
}

8 April 2005

\begin{abstract}
We consider the effect of weak uncorrelated quenched disorder (point defects) on a strongly fluctuating flux-line liquid. We use a hydrodynamic model which is based on mapping the flux-line system onto a quantum liquid of relativistic charged bosons in $2+1$ dimensions [P. Benetatos and M. C. Marchetti, Phys. Rev. B 64, 054518, (2001)]. In this model, flux lines are allowed to be arbitrarily curved and can even form closed loops. Point defects can be scalar or polar. In the latter case, the direction of their dipole moments can be random or correlated. Within the Gaussian approximation of our hydrodynamic model, we calculate disorder-induced corrections to the correlation functions of the flux-line fields and the elastic moduli of the flux-line liquid. We find that scalar disorder enhances loop nucleation, and polar (magnetic) defects decrease the tilt modulus.
\end{abstract}

Keywords: Superconductivity; Flux-line liquids; Quenched disorder; Point defects; Relativistic bosons

PACS: 74.25.Qt, 74.72-h 


\section{Introduction}

The physics of vortex-line arrays in type-II superconductors has been the subject of intense research activity in the past fifteen odd years [1, 2, 3, 4, 5]. Potential technological applications of high-temperature superconductors rely on the pinning of the vortices in order to eliminate dissipative losses from their motion. In addition, the competition between thermal fluctuations, intervortex interactions, and various types of disorder gives rise to a variety of phases, thus making vortex matter an excellent laboratory for the study of fundamental statistical mechanics.

The effect of disorder on the Abrikosov lattice phase of vortex arrays has been the subject of extensive theoretical and experimental studies $[6,7,8,9$, 10, 11]. Much less understood is the role of disorder in the vortex liquid phase, which is known to occupy a broad region of the magnetic-field-temperature phase diagram in high-temperature superconductors. A powerful method for describing both clean and disordered vortex liquids is the mapping of the statistical mechanics of directed line liquids in three dimensions onto that of two-dimensional nonrelativistic quantum bosons $[12,13,14]$. This mapping assumes small fluctuations of the flux lines away from the direction $\hat{\mathbf{z}}$ of the external applied field and intervortex interactions only among line segments at the same "height", $z$. The vortex lines map onto to the world lines of the quantum particles and this mapping is particularly useful for describing the effect of columnar pins caused by heavy-ion irradiation, which correspond to quenched impurities for the bosons [15]. In contrast, point defects in the superconductor correspond to time-dependent disorder for the bosons and are more difficult to treat in the quantum system. It has been shown, however, that this type of disorder yields "Lorentzian-squared" corrections to the thermal static structure function of the vortex liquid [16, 17] and does not renormalize the tilt modulus in the thermodynamic limit [17].

A more convenient framework for describing point disorder is the hydrodynamic description of directed flux-line liquids developed some time ago by M. C. Marchetti and D. R. Nelson [18, 19]. This earlier implementation of vortex liquid hydrodynamics takes into account the repulsion among flux-line segments at different heights, $z$, which renders the intervortex interaction nonlocal, but is restricted to directed lines, excluding overhangs or spontaneous vortex loops.

It was argued recently that vortex loops may be important in the vor- 
tex liquid phase, especially at low fields, where diverging loop fluctuations may destroy the coherence of the vortex state via a so-called "loop blow-out" transition [20, 21, 22]. Although earlier numerical evidence for such a transition in the uniformly frustrated $X Y$ model [22] was subsequently shown to be associated with boundary effects [23], these simulations do indicate that spontaneous vortex loop formation is important in vortex liquids. Also, recent experiments in YBCO show evidence of a liquid-to-liquid transition which appears to agree with the "loop blow-out" scenario [24]. Disorder, which is ubiquitous in real high-temperature materials, was not included in the above mentioned simulations. This renders a detailed comparison with the experiment problematic.

In this paper we present a hydrodynamic model of strongly fluctuating flux liquids that allows us to describe in a unified manner both field-induced lines and spontaneous loops. The key ingredient is a new scalar field that describes the density of flux-line length regardless of its direction or orientation and therefore incorporates the contribution from neighboring antiparallel segments associated with closed loos. We use this framework to study the effect of point disorder on the flux-line liquid by evaluating perturbatively disorder-induced corrections to the static correlation functions and to the elastic constants. Both "scalar" point disorder (that couples to flux lines irrespective of their orientation) and "vector" point disorder (arising from impurities carrying magnetic moments) can naturally be introduced in the model and play qualitatively different roles in renormalizing the elastic properties of the liquid. In particular, "scalar" disorder softens the single-line energy, enhancing the formation of vortex loops.

The statistical mechanics of strongly fluctuating vortex liquids in zero external magnetic field has been described theoretically by M. Kiometzis et al. [25] and by Z. Tešanović [20, 21] by mapping the vortex line system onto a collection of relativistic two-dimensional quantum bosons. In this mapping spontaneous vortex loop formation corresponds to particle-antiparticle creation and annihilation. Building on this work and its generalization to vortex liquids in a field, we recently proposed a general mapping of flux-line liquids of directed field-induced lines and spontaneous flux loops onto a twodimensional system of relativistic charged quantum bosons, where the external applied field for the bosons corresponds to the bosonic chemical potential [26]. The main difference between our model and earlier ones [20, 21, 22] is that, while these authors consider fluctuating vortices in a frozen magnetic 
field (corresponding to the limit of infinite penetration depth), we describe magnetic flux lines fluctuating in concert with their vortex cores by keeping the London penetration depth finite. By coarse-graining the boson model, we obtained a generalized hydrodynamic description of strongly fluctuating liquids of flux lines and loops.

After describing the model in Section 2, we use a Gaussian approximation of our generalized hydrodynamic free energy to evaluate the renormalization of the liquid properties from three different types of uncorrelated disorder in Sections 3, 4 and 5. Finally, we summarize in Section 6.

\section{The model}

In the hydrodynamic description of a directed flux-line liquid proposed by Marchetti and Nelson [18, 19], the large scale properties of the system are described in terms of the three components of a coarse-grained vector field, $\mathbf{t}(\mathbf{r})=\left\{\mathbf{t}_{\perp}, t_{z}\right\}$. The component $t_{z}$ is the areal density of flux lines along the direction $z$ of the external field piercing the $x y$ plane and directed along $\hat{\mathbf{z}}$. It is proportional to the $z$ component of the local magnetic field in the material. The two-dimensional vector field $\mathbf{t}_{\perp}$ describes the local tilt away from the $z$ direction and is proportional to the local magnetic field in the $x y$ plane. The correspondence between this hydrodynamic model and the familiar nonrelativistic boson model is well understood. It is important to note that the energy of a collection of flux lines in an applied field has full three-dimensional rotational symmetry. This symmetry is reduced in the nonrelativistic boson model which is invariant under Galilean boosts, corresponding to a uniform tilt of the flux lines away from the direction of the applied field. In the conventional hydrodynamic free energy, the single-line part is invariant under uniform tilt (Galilean symmetry), while the intervortex interaction has full rotational invariance in three dimensions. Full rotational invariance of the flux-line system corresponds to Lorentz invariance of the bosons, indicating that the quantum particles should be treated relativistically.

In Ref. [26], we showed that a three-dimensional liquid of directed fieldinduced lines and spontaneous flux loops can be mapped onto a $(2+1)$ dimensional liquid of relativistic charged bosons interacting via a screened electromagnetic field. We exploited this mapping to obtain a general hydrodynamic description of strongly fluctuating vortex liquids that allows for 
overhangs, loop formation, and nonlocal (in $z$ ) interaction among vortex segments at different "heights". The hydrodynamic description of strongly fluctuating vortex liquids is in terms of four coarse-grained fields: the three components of the vector field, $\mathbf{t}(\mathbf{r})=\left\{\mathbf{t}_{\perp}, t_{z}\right\}$, which measures the local magnetic field in the material, and an additional scalar field, $\rho(\mathbf{r})$. The new scalar field $\rho(\mathbf{r})$ appears because of the possibility of spontaneous loop formation. It measures the total contour length of flux lines enclosed in a unit volume centered at point $\mathbf{r}$, regardless of their direction or orientation. Upon coarsegraining, configurations of antiparallel neighboring flux lines, as arising from the spontaneous formation of a vortex loop, do not contribute to $\mathbf{t}(\mathbf{r})$, but do contribute to $\rho(\mathbf{r})$. In the relativistic boson mapping, $\mathbf{t}(\mathbf{r})=\left\{\mathbf{t}_{\perp}(\mathbf{r}), t_{z}(\mathbf{r})\right\}$ corresponds to the conserved current-charge-density, which is a $(2+1)$-vector with the time-like component $t_{z}$ the conventional charge density of bosons and $\mathbf{t}_{\perp}$ the charge-current density. In contrast to the charge density, $t_{z}$, the number density of bosons is not conserved due to creation-annihilation events (corresponding to vortex loop formation in the flux-line system). The scalar field $\rho(\mathbf{r})$ corresponds to the square of the amplitude of the complex boson field, $\Phi(\mathbf{r}) \equiv \sqrt{\rho(\mathbf{r})} \exp [i \theta(\mathbf{r})]$. The correlator $\left\langle\Phi^{*}\left(\mathbf{r}^{\prime}\right) \Phi(\mathbf{r})\right\rangle$ is proportional to the probability of finding a flux line segment connecting the two points, $\mathbf{r}$ and $\mathbf{r}^{\prime}[27]$, irrespective of the segment's orientation.

The hydrodynamic free energy functional of a strongly fluctuating fluxline liquid with disorder can be written as $\mathcal{F}=\mathcal{F}_{0}+\mathcal{F}_{D}$, where $\mathcal{F}_{0}$ is the free energy of the pure system and $\mathcal{F}_{D}$ incorporates the coupling to point defects. The pure contribution for a line liquid in an external field, $\mathbf{H}=H_{0} \hat{\mathbf{z}}$, is [26]

$$
\begin{aligned}
\mathcal{F}_{0}[\mathbf{t}, \rho]= & \int_{\mathbf{r}}\left\{\frac{\epsilon_{1}}{2} \rho(\mathbf{r})+\frac{\left(k_{B} T\right)^{2}}{8 \epsilon_{1} \rho(\mathbf{r})}[\nabla \rho(\mathbf{r})]^{2}+\frac{\epsilon_{1}}{2 \rho(\mathbf{r})}|\mathbf{t}(\mathbf{r})|^{2}-\frac{\phi_{0}}{4 \pi} \mathbf{H} \cdot \mathbf{t}(\mathbf{r})\right\} \\
& +\frac{1}{2 \Omega L} \sum_{\mathbf{q}} V(q)|\mathbf{t}(\mathbf{q})|^{2}
\end{aligned}
$$

where $\epsilon_{1}=\epsilon_{0} \ln \kappa$ is the bare flux-line tension, $\epsilon_{0}=\phi_{0}^{2} /(4 \pi \tilde{\lambda})^{2}, \tilde{\lambda}$ is the effective London penetration depth, and $\kappa \equiv \tilde{\lambda} / \xi$ is the Ginzburg-Landau parameter, with $\xi$ the coherence length. The last term (written in Fourier space) describes the intervortex interaction, with $V(q)=4 \pi \epsilon_{0} \tilde{\lambda}^{2} /\left(1+q^{2} \tilde{\lambda}^{2}\right)$. The average equilibrium values of the hydrodynamic fields are: $\mathbf{t}_{\perp 0}=0$, $t_{z 0}= \pm \rho_{0}$, and $\rho_{0}=\left(\left|H_{0}\right|-H_{c 1}\right) / \phi_{0}$ for $\left|H_{0}\right|>H_{c 1}$, while $\rho_{0}=0$ for $\left|H_{0}\right| \leq H_{c 1}$. Here $H_{c 1}=4 \pi \epsilon_{1} / \phi_{0}$ is the lower critical field and $\rho_{0}=1 / a_{0}^{2}$ 
represents the average areal density of flux lines in the $x y$ plane, with $a_{0}$ the intervortex spacing. Averages over the conformations of flux lines and flux loops are evaluated with the Boltzmann weight $\sim \exp \left[-\mathcal{F} / k_{B} T\right]$ and the constraint

$$
\nabla \cdot \mathbf{t}=0
$$

which prohibits flux lines from starting or ending within the sample. In the relativistic boson mapping it corresponds to charge conservation. In this paper we present the hydrodynamics of vortex liquids in isotropic superconductors, but the generalization to the anisotropic case is straightforward [26].

In contrast with the hydrodynamics of directed flux-line liquids, the effective free energy of Eq. (1) respects the rotational invariance of a pure flux-line system in an external field. This rotational invariance corresponds to the Lorentz invariance of the relativistic boson liquid. The non-Gaussian hydrodynamic free energy of directed vortex liquids [28] is obtained from Eq. (1) simply by setting $\rho(\mathbf{r}) \equiv t_{z}(\mathbf{r})$. This suggests that the difference

$$
t_{z}^{L}(\mathbf{r}) \equiv t_{z}(\mathbf{r})-\rho(\mathbf{r})
$$

may be interpreted as a density of lines associated with spontaneous vortex loop fluctuations.

\section{Point defects with magnetic moments par- allel to the external field}

Uncorrelated quenched disorder from point material defects is usually mod-

eled by a random field which couples to the fluctuations in $t_{z}(\mathbf{r})$ [16]. The disorder contribution to the free energy is written as

$$
\mathcal{F}_{D}=\int d^{2} r_{\perp} d z V_{D}(\mathbf{r}) \delta t_{z}(\mathbf{r})
$$

The quenched random potential is taken to be Gaussian, statistically homogeneous and isotropic, with $\overline{V_{D}(\mathbf{r})}=0$ and $\overline{V_{D}(\mathbf{r}) V_{D}\left(\mathbf{r}^{\prime}\right)}=\Delta \delta\left(\mathbf{r}-\mathbf{r}^{\prime}\right)$, where the overbar denotes the quenched average over disorder. A random field that couples to $t_{z}$ corresponds to quenched magnetic dipoles of random strength and position, with magnetic moments aligned with the $z$ direction. It favors 
local alignment of the flux lines with the direction of the external field (when $\left.V_{D}(\mathbf{r})<0\right)$. It corresponds to a random chemical potential for the nonrelativistic bosons [17], and to a random scalar potential coupling to the charge density for relativistic charged bosons [26].

In order to calculate the disorder-induced contributions to the various correlation functions characterizing the vortex liquid, we expand the fluxliquid free energy of Eq. (1) to quadratic order in the fluctuations of the fields from their equilibrium values, $\delta \mathbf{t}(\mathbf{r})=\mathbf{t}(\mathbf{r})-\mathbf{t}_{0}$ and $\delta \rho(\mathbf{r})=\rho(\mathbf{r})-\rho_{0}$. The resulting Gaussian approximation is given by

$$
\begin{aligned}
\mathcal{F}^{G}[\delta \mathbf{t}, \delta \rho] \approx & F^{\prime}+\frac{1}{2 \Omega L} \sum_{\mathbf{q}}\left\{\left[\frac{\epsilon_{1}}{\rho_{0}}+\frac{\left(k_{B} T\right)^{2}}{4 \epsilon_{1} \rho_{0}} q^{2}\right]|\delta \rho(\mathbf{q})|^{2}+\left[\frac{\epsilon_{1}}{\rho_{0}}+V(q)\right]|\delta \mathbf{t}(\mathbf{q})|^{2}\right. \\
& \left.-\frac{\epsilon_{1}}{\rho_{0}}\left[\delta t_{z}(\mathbf{q}) \delta \rho(-\mathbf{q})+\delta t_{z}(-\mathbf{q}) \delta \rho(\mathbf{q})\right]\right\}+\mathcal{F}_{D}
\end{aligned}
$$

where $F^{\prime}$ is the equilibrium value. The Gaussian approximation to our hydrodynamic model for the pure system can be viewed as a modified OrnsteinZernike theory for flux lines [29]. The familiar Gaussian free energy of directed lines is obtained by setting $\delta \rho(\mathbf{q}) \equiv \delta t_{z}(\mathbf{q})$ in Eq. (5) and neglecting terms of higher order in $q$, with the result

$$
\mathcal{F}_{d}^{G}[\delta \mathbf{t}] \approx F^{\prime}+\frac{1}{2 \Omega L \rho_{0}^{2}} \sum_{\mathbf{q}}\left\{c_{44}^{0}(q)\left|\delta \mathbf{t}_{\perp}(\mathbf{q})\right|^{2}+c_{11}^{0}(q)\left|\delta t_{z}(\mathbf{q})\right|^{2}\right\}+\mathcal{F}_{D}
$$

where

$$
\begin{aligned}
& c_{44}^{0}(q)=\rho_{0} \epsilon_{1}+\rho_{0}^{2} V(q), \\
& c_{11}^{0}(q)=\rho_{0}^{2} V(q),
\end{aligned}
$$

are the bare local tilt and compressional moduli, respectively, of a pure $d i$ rected flux-line liquid.

The correlation functions of the fluctuations of the hydrodynamic fields are immediately evaluated by carrying out both a thermal average with weight $\sim \exp \left[-\mathcal{F}^{G} / k_{B} T\right]$ and an average over quenched disorder. Retaining here and below only the first-order corrections in $\Delta$, we obtain

$$
\overline{\left\langle\delta t_{z}(\mathbf{q}) \delta t_{z}(-\mathbf{q})\right\rangle_{G}}=k_{B} T \frac{\rho_{0} q_{\perp}^{2}}{D(\mathbf{q})}+\Delta\left[\frac{\rho_{0} q_{\perp}^{2}}{D(\mathbf{q})}\right]^{2}
$$




$$
\begin{aligned}
\overline{\left\langle t_{\perp i}(\mathbf{q}) t_{\perp j}(-\mathbf{q})\right\rangle_{G}}= & k_{B} T\left[\frac{\rho_{0}^{2} k_{B} T}{c_{44}^{0}(q)} P_{i j}^{T}\left(\mathbf{q}_{\perp}\right)+\frac{\rho_{0} q_{z}^{2}}{D(\mathbf{q})} P_{i j}^{L}\left(\mathbf{q}_{\perp}\right)\right] \\
& +\Delta\left[\frac{\rho_{0} q_{\perp} q_{z}}{D(\mathbf{q})}\right]^{2} P_{i j}^{L}\left(\mathbf{q}_{\perp}\right), \\
\frac{\langle\delta \rho(\mathbf{q}) \delta \rho(-\mathbf{q})\rangle_{G}}{=} & k_{B} T \frac{\rho_{0} q^{2}}{D(\mathbf{q})} \frac{c_{44}^{0}(q)}{\rho_{0} \epsilon_{1}\left(1+q^{2} a_{0}^{2}\left(a_{0} / 2 l_{z}\right)^{2}\right)} \\
& +\Delta\left[\frac{\rho_{0} q_{\perp}^{2}}{D(\mathbf{q})\left(1+q^{2} a_{0}^{2}\left(a_{0} / 2 l_{z}\right)^{2}\right)}\right]^{2},
\end{aligned}
$$

where $i, j=(x, y), P_{i j}^{L}\left(\mathbf{q}_{\perp}\right)=q_{\perp i} q_{\perp j} / q_{\perp}^{2}$ and $P_{i j}^{T}\left(\mathbf{q}_{\perp}\right)=\delta_{i j}-P_{i j}^{L}\left(\mathbf{q}_{\perp}\right)$ are longitudinal and transverse projection operators and

$$
D(\mathbf{q}) \equiv q_{z}^{2} c_{44}^{0}(q) / \rho_{0}+q_{\perp}^{2}\left[c_{11}^{0}(q)+\delta c_{11}(q)\right] / \rho_{0} .
$$

As seen below, $\delta c_{11}(q)$ represents a short scale correction to the compressional modulus. It is given by

$$
\delta c_{11}(q)=\rho_{0} \epsilon_{1} \frac{q^{2} a_{0}^{2}\left(a_{0} / 2 l_{z}\right)^{2}}{1+q^{2} a_{0}^{2}\left(a_{0} / 2 l_{z}\right)^{2}},
$$

with $l_{z}=\epsilon_{1} a_{0}^{2} /\left(2 k_{B} T\right)$. The autocorrelation function of the longitudinal part of $\mathbf{t}_{\perp}$ is simply related to that of $t_{z}$ as the "continuity" equation (2) requires $\mathbf{q}_{\perp} \cdot \mathbf{t}_{\perp}=-q_{z} t_{z}$. Cross correlations among the fields are also not vanishing but will not be given here.

Compressional and tilt deformations of the line liquid can be described by defining effective local (wavector-dependent) elastic moduli as

$$
\begin{gathered}
\frac{\rho_{0}^{2} k_{B} T}{c_{11}\left(q_{\perp}\right)}=\lim _{q_{z} \rightarrow 0} \overline{\left\langle\delta t_{z}(\mathbf{q}) \delta t_{z}(-\mathbf{q})\right\rangle}, \\
\frac{\rho_{0}^{2} k_{B} T}{c_{44}\left(q_{z}\right)}=\lim _{q_{\perp} \rightarrow 0} P_{i j}^{T}\left(\mathbf{q}_{\perp}\right) \overline{\left\langle t_{i}(\mathbf{q}) t_{j}(-\mathbf{q})\right\rangle} .
\end{gathered}
$$

In the absence of disorder, this gives $c_{11}\left(q_{\perp}\right)=c_{11}^{0}\left(q_{\perp}\right)+\delta c_{11}\left(q_{\perp}\right)$, while $c_{44}\left(q_{\perp}\right)=c_{44}^{0}\left(q_{\perp}\right)$ is unchanged. The length scale $l_{z}$ is the thermally-induced entanglement correlation length introduced by Nelson [12]. A single flux line at finite temperature wanders transversally as it crosses the sample along 
the $z$ direction. The entanglement length $l_{z}$ represents a "collision" length in the time-like variable $z$. It is the length scale a single flux line travels in the $z$ direction before wandering a transverse distance of order $a_{0}$ and colliding with a neighbor. Such "collisions" tend to stiffen the compressional modulus on small length scales (of order $a_{0}$ ) due to the caging of each line by its neighbors.

The long-wavelength disorder-renormalized elastic constants are defined as

$$
\begin{aligned}
& \frac{\rho_{0}^{2} k_{B} T}{c_{11}^{R}}=\lim _{q_{\perp} \rightarrow 0} \lim _{q_{z} \rightarrow 0} \overline{\left\langle\delta t_{z}(\mathbf{q}) \delta t_{z}(-\mathbf{q})\right\rangle} \\
& \frac{\rho_{0}^{2} k_{B} T}{c_{44}^{R}}=\lim _{q_{z} \rightarrow 0} \lim _{q_{\perp} \rightarrow 0} P_{i j}^{T}\left(\mathbf{q}_{\perp}\right) \overline{\left\langle t_{i}(\mathbf{q}) t_{j}(-\mathbf{q})\right\rangle}
\end{aligned}
$$

where the order of the limits is important. These definitions are consistent with the definition of the elastic constants as response functions. Within the Gaussian approximation, point disorder described as a random field coupled to $t_{z}(\mathbf{r})$ softens the compressional modulus, but it does not renormalize the tilt modulus,

$$
\begin{aligned}
c_{11}^{R} & =\frac{c_{11}^{0}(0)}{1+\Delta \rho_{0}^{2} /\left(c_{11}^{0}(0) k_{B} T\right)}, \\
c_{44}^{R} & =c_{44}^{0}(0) .
\end{aligned}
$$

As discussed in Ref. [26], the long-wavelength limit of the autocorrelation function of the fluctuations in the field $t_{z}^{L}(\mathbf{r})$, associated with spontaneous vortex loops, determines the renormalized single-line energy $\epsilon_{1}$, according to

$$
\lim _{q_{\perp} \rightarrow 0} \lim _{q_{z} \rightarrow 0} \overline{\left\langle\delta t_{z}^{L}(\mathbf{q}) \delta t_{z}^{L}(-\mathbf{q})\right\rangle_{G}}=\frac{\rho_{0} k_{B} T}{\epsilon_{1}^{R}},
$$

irrespective of the order of limits. A random field that couples to $t_{z}$ gives no corrections to the line energy and $\epsilon_{1}^{R}=\epsilon_{1}$. It is important to stress the difference between the line energy, $\epsilon_{1}$, and the line tilt stiffness, $\tilde{\epsilon_{1}}$. The former is the energy per unit length needed to create a flux line in the direction of the external applied field. It is related to the lower critical field and, in the boson analogy, it corresponds to the boson rest mass. The tilt coefficient $\tilde{\epsilon_{1}}$ measures the stiffness of a line against a tilt away from the direction of 
the external field. In clean, isotropic superconductors, $\epsilon_{1}=\tilde{\epsilon_{1}}$ are equal, but they can differ strongly in anisotropic materials [30]. Using the free energy of strongly fluctuating flux-line liquids in uniaxial anisotropic superconductors [26] (where the external field is applied along the $c$-axis of the material), one can show that the long-wavelength limit of autocorrelator of the $t_{z}^{L}$ field yields $\epsilon_{1}$, while the single-vortex part of the tilt modulus yields $\tilde{\epsilon_{1}}$. In the following sections, we shall show that different types of disorder have different effect on these two distinct quantities.

Finally, the disorder-induced correction to the autocorrelators of the two different densities, Eqs. (9) and (11), differ at finite wavevectors. This difference becomes pronounced in strongly entangled liquids (high temperature and/or density), where $a_{0} / l_{z} \sim 1\left(a_{0} / l_{z} \approx 0.3\right.$ for $T=77 K$ and $\left.B=2 T\right)$.

\section{Scalar point defects}

In this section we model uncorrelated point disorder as a scalar random field that couples to the scalar field $\rho(\mathbf{r})$,

$$
\mathcal{F}_{D}=\int d^{2} r_{\perp} d z V_{D}(\mathbf{r}) \delta \rho(\mathbf{r})
$$

This corresponds to a random contribution to the line stiffness $\epsilon_{1}$ (or to the quantum particles' mass in the relativistic charged boson mapping). The random field is assumed to have zero mean and correlations given by Eq. (??), although with a different disorder strength, $\Delta$. This random field physically represents uncorrelated pinning centers which favor the nucleation of flux lines irrespective of their orientation. We therefore expect that it will favor vortex loop formation. In a liquid of directed lines, there is no difference between this case and the one discussed in Section 3.

The thermal part of all correlation functions is, of course, unchanged. Introducing the notation $\overline{\delta\langle\ldots\rangle} \equiv \overline{\langle\ldots\rangle}-\langle\ldots\rangle$ to denote the contribution due to disorder to the various correlation functions, we find

$$
\begin{gathered}
\overline{\delta\left\langle\delta t_{z}(\mathbf{q}) \delta t_{z}(-\mathbf{q})\right\rangle_{G}}=\Delta\left[\frac{\rho_{0} q_{\perp}^{2}}{D(\mathbf{q})\left(1+q^{2} a_{0}^{2}\left(a_{0} / 2 l_{z}\right)^{2}\right)}\right]^{2}, \\
\overline{\delta\left\langle t_{i}(\mathbf{q}) t_{j}(-\mathbf{q})\right\rangle_{G}}=\Delta\left[\frac{\rho_{0} q_{\perp} q_{z}}{D(\mathbf{q})\left(1+q^{2} a_{0}^{2}\left(a_{0} / 2 l_{z}\right)^{2}\right)}\right]^{2} P_{i j}^{L}\left(\mathbf{q}_{\perp}\right),
\end{gathered}
$$




$$
\overline{\delta\langle\delta \rho(\mathbf{q}) \delta \rho(-\mathbf{q})\rangle_{G}}=\Delta\left[\frac{\rho_{0} q^{2}}{D(\mathbf{q})} \frac{c_{44}^{0}(q)}{\rho_{0} \epsilon_{1}\left(1+q^{2} a_{0}^{2}\left(a_{0} / 2 l_{z}\right)^{2}\right)}\right]^{2} .
$$

Again, disorder leaves the transverse part of the $\mathbf{t}_{\perp}$ correlations unchanged and therefore it does not renormalize the long-wavelength tilt modulus (in the Gaussian approximation). The renormalized long-wavelength compressional modulus is identical to that given in Eq. (18), although the two differ at finite wavelengths. This type of scalar disorder does, however, yield a finite renormalization of the line energy that determines vortex loop fluctuations. Using the definition given in Eq.(20), we find

$$
\frac{1}{\epsilon_{1}^{R}}=\frac{1}{\epsilon_{1}}+\frac{\Delta \rho_{0}}{k_{B} T \epsilon_{1}^{2}}
$$

The disorder-induced correction to the line energy on the right hand side of Eq. (25) can be written as the ratio of two length scales as $\epsilon_{1}^{R}=\epsilon_{1} /(1+$ $\left.l_{z} / l_{D}\right)$, where $l_{z}$ is the thermal entanglement length introduced earlier and $l_{D}=\epsilon_{1}^{2} /\left(2 \Delta \rho_{0}^{2}\right)$ is a characteristic entanglement length associated with disorder. This type of quenched disorder softens the line tension, enhancing the formation of vortex loops. The condition $l_{z} \approx l_{D}$ marks a crossover at $T \sim\left(\Delta / \epsilon_{1}\right) B_{z}$ from a regime where loop fluctuations are thermally dominated to one where they are induced by quenched-disorder. Although numerical studies of the pure uniformly frustrated $X Y$ model have ruled out the scenario of the "loop blow-out" transition proposed by Tešanović [20, 21] and by Sudbø and collaborators [22], our result indicates that vortex loop formation is enhanced by disorder, suggesting the possibility that such a transition may be recovered in sufficiently disordered systems, where the line tension may be driven to zero.

\section{Point defects with randomly oriented mag- netic moments}

Finally, in this section we consider uncorrelated point impurities that can be modeled as quenched magnetic dipoles with random orientation. This corresponds to a vector disorder potential that couples with statistically equal strength to all components of the local magnetic field, yielding

$$
\mathcal{F}_{D}=\int d^{2} r_{\perp} d z \mathbf{V}_{D}(\mathbf{r}) \cdot \mathbf{t}(\mathbf{r})
$$


with $\overline{V_{D \mu}(\mathbf{r})}=0$ and $\overline{V_{D \mu}(\mathbf{r}) V_{D \nu}\left(\mathbf{r}^{\prime}\right)}=\delta_{\mu \nu} \Delta \delta\left(\mathbf{r}-\mathbf{r}^{\prime}\right)$, for $\mu, \nu=(x, y, z)$. The random field favors alignment of the local magnetic field due to flux lines with uncorrelated quenched random directions.

To Gaussian order, the disorder-induced corrections to the correlation functions are

$$
\begin{gathered}
\overline{\delta\left\langle\delta t_{z}(\mathbf{q}) \delta t_{z}(-\mathbf{q})\right\rangle_{G}}=\Delta\left[\frac{\rho_{0} q_{\perp} q}{D(\mathbf{q})}\right]^{2}, \\
\overline{\delta\left\langle t_{i}(\mathbf{q}) t_{j}(-\mathbf{q})\right\rangle_{G}}=\Delta\left\{\left[\frac{\rho_{0}^{2}}{c_{44}^{0}(q)}\right]^{2} P_{i j}^{T}\left(\mathbf{q}_{\perp}\right)+\left[\frac{\rho_{0} q_{\perp} q}{D(\mathbf{q})}\right]^{2} P_{i j}^{L}\left(\mathbf{q}_{\perp}\right)\right\}, \\
\overline{\delta\langle\delta \rho(\mathbf{q}) \delta \rho(-\mathbf{q})\rangle_{G}}=\Delta\left[\frac{\rho_{0} q_{\perp} q}{D(\mathbf{q})\left(1+q^{2} a_{0}^{2}\left(a_{0} / 2 l_{z}\right)^{2}\right)}\right]^{2} .
\end{gathered}
$$

The disorder-induced correction to the oriented areal density autocorrelation, Eq (27), differs significantly from that obtained for the other two types of disorder when $q_{\perp} \approx 0$ and $q_{z} \neq 0$.

As in the case presented in Section 3, this type of disorder does not renormalize the line stiffness $\epsilon_{1}$. The transverse part of the tangent field autocorrelator does, however, acquire a finite correction, which leads to a renormalization of the long-wavelength tilt modulus. From Eq. (17), we find

$$
\frac{1}{c_{44}^{R}}=\frac{1}{c_{44}^{0}}+\frac{\Delta}{k_{B} T} \frac{1}{\left[\epsilon_{1}+\rho_{0} \phi_{0}^{2} /(4 \pi)\right]^{2}} .
$$

As shown by Larkin and Vinokur [31], the renormalization of $c_{44}$ can be related to that of its single-vortex part, $c_{44}^{v}=\rho_{0} \tilde{\epsilon}_{1}$, with $\tilde{\epsilon}_{1}$ the tilt stiffness of a directed line. This is renormalized by the "vector" disorder according to

$$
\tilde{\epsilon}_{1}^{R}=\epsilon_{1}\left(1-l_{z} / l_{D}\right) .
$$

Isotropic random dipoles tend to align flux-line segments along the random directions of the magnetic moments carried by the impurities and facilitate tilting away from the $z$-direction. On the other hand, the line tension defined in Eq. (20) and associated with loop fluctuations remains unrenormalized by this type of disorder. In both cases, the disorder-induced corrections 
are determined by the ratio $l_{z} / l_{D}$ of the thermal and disorder entanglement lengths (see Eq. (25)).

Finally, the disorder-induced renormalization of the compressional modulus is the same as for the other two types of point disorder in the longwavelength limit, although it differs at finite wavevectors.

\section{Conclusions}

In this paper, we have calculated weak-disorder-induced corrections to the static correlation functions of strongly fluctuating (arbitrarily curved and looping) flux-line liquids using a Gaussian hydrodynamic approach. Three realizations of point disorder have been considered, corresponding to magnetic and non-magnetic defects. Our results for the structure function, the scalar density $(\rho(\mathbf{r}))$ autocorrelator, and the tilt field $\left(\mathbf{t}_{\perp}(\mathbf{r})\right)$ correlations can, in principle, be probed experimentally using polarized and unpolarized neutron scattering. We have predicted qualitative differences between these quantities at finite wavevectors for different types of disorder which are experimentally testable. From these correlations, we have also obtained the disorder-induced corrections to the elastic moduli. The compressional modulus gets softened for all three types of point disorder. Loop fluctuations are enhanced by scalar disorder. The tilt modulus is softened by isotropic random polar (magnetic) disorder.

A softening of the elastic constants by quenched disorder may appear surprising, as naively one would expect pinning to stiffen the system. A result qualitatively similar to ours has, however, been obtained before in a different system [32]. Preliminary results of a calculation of the disorderinduced correction to the compressional modulus taking into account nonGaussian terms in the free energy suggest that this correction may change sign depending on the system parameters (temperature or magnetic field), indicating that a perturbative approach may not adequate in this case [33]. If the softening were to survive a non-perturbative calculation, this would signal a disorder-driven instability of the vortex liquid phase. Clearly more work beyond the Gaussian approximation is needed to answer these questions.

MCM was supported by the National Science Foundation under grant DMR-0305407. 


\section{References}

[1] G. Blatter, M. V. Feigel'man, V. B. Geshkenbein, A. I. Larkin, and V. M. Vinokur, Rev. Mod. Phys. 661125 (1994).

[2] E. H. Brandt, Pep. Prog. Phys. 581465 (1995).

[3] T. Nattermann and S. Scheidl, Adv. Phys. 49607 (2000).

[4] T. Giamarchi and S. Bhattacharya, in High Magnetic Fields: Applications to condensed matter physics and spectroscopy, C. Bertier et al., eds., p. 314 (Springer-Verlag, 2002).

[5] G. W. Crabtree and D. R. Nelson, Phys. Today 50(4), 38 (1997).

[6] A. I. Larkin and Y. N. Ovchinikov, J. Low Temp. Phys. 34, 409 (1979).

[7] T. Nattermann, Phys. Rev. Lett. 64, 2454 (1990).

[8] D. S. Fisher, M. P. A. Fisher, and D. A. Huse, Phys. Rev. B 43, 130 (1990).

[9] T. Giamarchi and P. Le Doussal, Phys. Rev. B 52, 1242 (1995).

[10] Y. Y. Goldschmidt, Phys. Rev. B 56, 2800 (1997).

[11] T. Klein et al., Nature 413, 404 (2001), and references therein.

[12] D. R. Nelson, Phys. Rev. Lett. 60, 1973 (1988).

[13] M. P. A. Fisher and D. H. Lee, Phys. Rev. B 39, 2756 (1989).

[14] D. R. Nelson and H. S. Seung, Phys. Rev. B 39, 9153 (1989).

[15] D. R. Nelson and V. N. Vinokur, Phys. Rev. B 48, 13060 (1993).

[16] D. R. Nelson and P. Le Doussal, Phys. Rev. B 42, 10113 (1990).

[17] U. C. Täuber and D. R. Nelson, Phys. Rep. 289, 157 (1997).

[18] M. C. Marchetti and D. R. Nelson, Phys. Rev. B 42, 9938 (1990).

[19] M. C. Marchetti and D. R. Nelson, Physica C 174, 40 (1991). 
[20] Z. Tešanović, Phys. Rev. B 51, 16304 (1995).

[21] Z. Tešanović, Phys. Rev. B 59, 6449 (1999).

[22] A. K. Nguyen and A. Sudbø, Phys. Rev. B 60, 15037 (1999).

[23] P. Olsson and S. Teitel, Phys. Rev. B 67, 144514 (2003).

[24] F. Bouquet et al., Nature 411, 448 (2001).

[25] M. Kiometzis, H. Kleinert, and A. M. J. Schakel, Fortschr. Phys. 43, 697 (1995).

[26] P. Benetatos and M. C. Marchetti, Phys. Rev. B 64, 054518 (2001).

[27] H. Kleinert, Gauge Fields in Condensed Matter, (World Scientific, Singapore, 1989).

[28] P. Benetatos and M. C. Marchetti, Physica C 332, 237 (2000).

[29] L. S. Ornstein and F. Zernike, Proc. Acad. Sci. Amst. 17, 793 (1914).

[30] V. G. Kogan, Phys. Rev. B 24, 1572 (1981).

[31] A. I. Larkin and V. M. Vinokur, Phys. Rev. Lett. 75, 4666 (1995).

[32] L. Radzihovsky and D. R. Nelson, Phys. Rev. A 44, 3525 (1991).

[33] P. Benetatos and M. C. Marchetti (unpublished). 